\title{
Structure of Rademacher subspaces in Cesàro type spaces
}

by

\author{
Sergey V. Astashkin (Samara) and Lech Maligranda (Luleå)
}

Dedicated to the memory of Aleksander Petczyński (2 July 1932 - 20 December 2012)

\begin{abstract}
The structure of the closed linear span $\mathcal{R}$ of the Rademacher functions in the Cesàro space $\mathrm{Ces}_{\infty}$ is investigated. It is shown that every infinite-dimensional subspace of $\mathcal{R}$ either is isomorphic to $l_{2}$ and uncomplemented in $\mathrm{Ces}_{\infty}$, or contains a subspace isomorphic to $c_{0}$ and complemented in $\mathcal{R}$. The situation is rather different in the $p$-convexification of $\mathrm{Ces}_{\infty}$ if $1<p<\infty$.
\end{abstract}

1. Introduction. The behaviour of the Rademacher functions in the spaces $L_{p}=L_{p}[0,1]$ is well known. By the classical Khintchine inequality, there exists a constant $A_{p}>0$ such that for all real numbers $a_{k}, k=1,2, \ldots$, we have

$$
A_{p}^{-1}\left(\sum_{k=1}^{\infty} a_{k}^{2}\right)^{1 / 2} \leq\left\|\sum_{k=1}^{\infty} a_{k} r_{k}\right\|_{L_{p}} \leq A_{p}\left(\sum_{k=1}^{\infty} a_{k}^{2}\right)^{1 / 2}, \quad 0<p<\infty,
$$

that is, $\left\{r_{n}\right\}_{n=1}^{\infty}$ spans an isomorphic copy of $l_{2}$ in $L_{p}$ for every $0<p<\infty$. Moreover, the subspace $\left[r_{n}\right]$ is complemented in $L_{p}$ for $1<p<\infty$, and not complemented in $L_{1}$ since no complemented infinite-dimensional subspace of $L_{1}$ can be reflexive. Moreover, $\left\|\sum_{k=1}^{n} a_{k} r_{k}\right\|_{L_{\infty}[0,1]}=\sum_{k=1}^{n}\left|a_{k}\right|$, and so the Rademacher functions span in $L_{\infty}$ an isometric copy of $l_{1}$, which is known to be uncomplemented (see [13, Theorem 2.b.4(ii)], [15, Theorem 1] and [2, Theorem 3.4]).

Investigations of Rademacher sums in the Cesàro function spaces $\operatorname{Ces}_{p}:=$ $\operatorname{Ces}_{p}[0,1]$ were initiated in [6]. The Cesàro spaces consist of all Lebesgue

2010 Mathematics Subject Classification: Primary 46E30, 46B20; Secondary 46B42.

Key words and phrases: Rademacher functions, Cesàro function space $\mathrm{Ces}_{\infty}$, KorenblyumKreĬn-Levin space, Cesàro function spaces $K_{p}$, subspaces, complemented subspaces. 
measurable real-valued functions $f$ on $[0,1]$ such that

$$
\|f\|_{\operatorname{Ces}_{p}}=\left[\int_{0}^{1}\left(\frac{1}{x} \int_{0}^{x}|f(t)| d t\right)^{p} d x\right]^{1 / p}<\infty \quad \text { for } 1 \leq p<\infty
$$

and

$$
\|f\|_{\operatorname{Ces} \infty}=\sup _{0<x \leq 1} \frac{1}{x} \int_{0}^{x}|f(t)| d t<\infty \quad \text { for } p=\infty .
$$

The latter space $\mathrm{Ces}_{\infty}$ appeared already in 1948 [10] (see also [14, [17, p. 469] and [16, p. 26]) and is known as the Korenblyum-Krein-Levin space K.

Further, we will also consider the $p$-convexification of the space $K=$ $\operatorname{Ces}_{\infty}, 1<p<\infty$, which will be denoted by $K_{p}$, consisting of all Lebesgue measurable real-valued functions $f$ on $[0,1]$ such that the norm

$$
\|f\|_{K_{p}}=\sup _{0<x \leq 1}\left(\frac{1}{x} \int_{0}^{x}|f(t)|^{p} d t\right)^{1 / p}
$$

is finite.

The Cesàro function spaces $\operatorname{Ces}_{p}, 1 \leq p \leq \infty$, are not rearrangement invariant, and are not isomorphic to $L_{q}$-spaces for any $1 \leq q \leq \infty$ (see [5], 7] and [8], where also other properties are investigated). However, similarly to $L_{p}$-spaces, there is also an essential difference in the behaviour of Rademacher sums in $\operatorname{Ces}_{p}$ for $1 \leq p<\infty$ and $p=\infty$. Namely, as proved in [6], for any $1 \leq p<\infty$, the sequence $\left\{r_{n}\right\}_{n=1}^{\infty}$ is equivalent in $\operatorname{Ces}_{p}$ to the unit vector basis of $l_{2}$, i.e.,

$$
B_{p}^{-1}\left(\sum_{k=1}^{\infty} a_{k}^{2}\right)^{1 / 2} \leq\left\|\sum_{k=1}^{\infty} a_{k} r_{k}\right\|_{\operatorname{Ces}_{p}} \leq B_{p}\left(\sum_{k=1}^{\infty} a_{k}^{2}\right)^{1 / 2}
$$

for a suitable constant $B_{p}>0$ and for all real $a_{k}, k=1,2, \ldots$ On the other hand, we have

$$
\begin{aligned}
& C_{p}^{-1}\left[\left(\sum_{k=1}^{\infty} a_{k}^{2}\right)^{1 / 2}+\sup _{m \in \mathbb{N}}\left|\sum_{k=1}^{m} a_{k}\right|\right] \leq\left\|\sum_{k=1}^{\infty} a_{k} r_{k}\right\|_{K_{p}} \\
& \leq C_{p}\left[\left(\sum_{k=1}^{\infty} a_{k}^{2}\right)^{1 / 2}+\sup _{m \in \mathbb{N}}\left|\sum_{k=1}^{m} a_{k}\right|\right],
\end{aligned}
$$

with some constant $C_{p}>0$ which depends only on $p \in[1, \infty)$. In particular, $\sum_{k=1}^{\infty} a_{k} r_{k}$ converges in $K_{p}$ if and only if both $\sum_{k=1}^{\infty} a_{k}^{2}$ and $\sum_{k=1}^{\infty} a_{k}$ are convergent. Moreover, (1.1) shows that the Rademacher functions form a conditional basis in the subspace

$$
\mathcal{R}_{p}:=\left[r_{k}\right] \text { spanned by } r_{k}, k=1,2, \ldots, \text { in } K_{p}, 1 \leq p<\infty .
$$


The aim of this paper is to describe the geometrical structure of the space $\mathcal{R}_{p}$ for $1 \leq p<\infty$. The following main results, which can be treated as a Kadec-Pełczyński type alternative for the Rademacher subspaces of $K_{p}$, indicate that their structures in the cases $p=1$ and $1<p<\infty$ are different.

THEOREM 1. Every infinite-dimensional subspace of $\mathcal{R}$ either is isomorphic to $l_{2}$ and uncomplemented in $K=\mathrm{Ces}_{\infty}$, or contains a subspace isomorphic to $c_{0}$ and complemented in $\mathcal{R}$.

TheOREM 2. Every infinite-dimensional subspace of $\mathcal{R}_{p}, 1<p<\infty$, either is isomorphic to $l_{2}$ and complemented in $K_{p}$, or contains a subspace isomorphic to $c_{0}$ and complemented in $\mathcal{R}_{p}$.

It is worth noting that comparing Theorem 2 with Leibov's results relating to the space of functions of bounded mean oscillation (see [11]) shows that the structures of the Rademacher subspaces in $K_{p}, 1<p<\infty$, and in BMO are similar. Generally speaking, this is not surprising, because Rademacher sums satisfy in BMO inequalities completely analogous to (1.1) (see also [4, where it is proved, among other results, that the subspace $\left[r_{k}\right]$ spanned by the Rademacher functions in BMO is not complemented in BMO). At the same time, it is instructive to emphasize the following point. In [11], Leibov uses the fact that the continuous embedding BMO $\hookrightarrow L_{1}$ factorizes through $L_{p}$ for any $p \in(1, \infty)$, which allows him to apply the well-known Kadec-Pełczyński result about complementability of any subspace of $L_{p}$, $p \geq 2$, isomorphic to $l_{2}$. However, by contrast, the continuous embedding $\mathrm{Ces}_{\infty} \hookrightarrow L_{p}$ holds if and only if $p=1$; hence we cannot now use the KadecPełczyński argument, and the result obtained (Theorem 1) essentially differs from the one proved by Leibov [11].

In what follows, given two positive functions (quasi-norms) $f$ and $g$ we write $f \asymp g$ if there exists a constant $C>0$ independent of all or of a part of parameters such that $C^{-1} f \leq g \leq C f$. As usual, we denote by $\left[x_{n}\right]$ the closed linear span of a sequence $\left\{x_{n}\right\}_{n=1}^{\infty}$ in a Banach space $X$, and set $\|f\|_{d}:=\left(\sum_{n=1}^{\infty} a_{n}^{2}\right)^{1 / 2}$ for a Rademacher sum $f=\sum_{n=1}^{\infty} a_{n} r_{n}$ converging a.e. on $[0,1]$. Moreover, we write $K_{1}=K$ and $\mathcal{R}_{1}=\mathcal{R}$.

The paper is organized as follows. In Section 2, based on some constructions from [11] and [4, we study properties of block bases of the Rademacher system in Cesàro type spaces. We show that, depending on whether $\liminf _{n \rightarrow \infty}\left\|u_{n}\right\|_{d}>0$ or $\liminf _{n \rightarrow \infty}\left\|u_{n}\right\|_{d}=0$, a block basis $\left\{u_{n}\right\}_{n=1}^{\infty}$ of the Rademacher functions weakly converging to zero in $K_{p}, 1 \leq p<\infty$, and such that $C^{-1} \leq\left\|u_{n}\right\|_{K_{p}} \leq C$ for some $C>0$ and all $n \in \mathbb{N}$ contains a subsequence equivalent to the unit vector basis of $l_{2}$ or $c_{0}$ (Theorem 3 ). This allows us to prove, in Theorem 4, that for each $1 \leq p<\infty$ every 
infinite-dimensional subspace of $\mathcal{R}_{p}$ either is isomorphic to $l_{2}$, or contains a subspace isomorphic to $c_{0}$ and complemented in $\mathcal{R}_{p}$.

In Sections 3 and 4, we complete the proof of Theorems 1 and 2 by exhibiting an essential difference in the geometrical structure of subspaces of $\mathcal{R}_{p}$ in the cases $p=1$ and $1<p<\infty$. Finally, in Section 5, we finish with some remarks relating to a more general weighted version of the Cesàro space.

2. Block bases of the Rademacher system in $K_{p}, 1 \leq p<\infty$. Let $\left\{u_{n}\right\}_{n=1}^{\infty}$ be a block basis of the Rademacher system $\left\{r_{k}\right\}_{k=1}^{\infty}$, that is,

$$
u_{n}=\sum_{k=m_{n}+1}^{m_{n+1}} a_{k} r_{k}, \quad 0<m_{1}<m_{2}<\cdots, n \in \mathbb{N} .
$$

THeOrem 3. Let $1 \leq p<\infty$, and $\left\{u_{n}\right\}_{n=1}^{\infty}$ be a block basis of $\left\{r_{k}\right\}_{k=1}^{\infty}$ weakly converging to zero in $K_{p}$ with $1 / c_{0} \leq\left\|u_{n}\right\|_{K_{p}} \leq c_{0}$ for some constant $c_{0}>0$ and for all $n \in \mathbb{N}$.

(a) If there is $\varepsilon>0$ such that $\left\|u_{n}\right\|_{d} \geq \varepsilon(n \in \mathbb{N})$, then $\left\{u_{n}\right\}_{n=1}^{\infty}$ contains a subsequence equivalent to the unit vector basis of $l_{2}$.

(b) If $\left\|u_{n}\right\|_{d} \rightarrow 0$ as $n \rightarrow \infty$, then $\left\{u_{n}\right\}_{n=1}^{\infty}$ contains a subsequence equivalent to the unit vector basis of $c_{0}$.

To prove Theorem 3 we will need some auxiliary facts. First, we observe that $\left\{r_{k}\right\}_{k=1}^{\infty}$ is not weakly convergent to zero in $K_{p}$. In fact, let $\varphi_{0}$ be a linear functional defined on the linear span of $r_{k}, k=1,2, \ldots$, by

$$
\varphi_{0}\left(\sum_{k=1}^{n} a_{k} r_{k}\right)=\sum_{k=1}^{n} a_{k}
$$

where $n \in \mathbb{N}, a_{k} \in \mathbb{R}, k=1, \ldots, n$. By 1.1, we have

$$
\left|\varphi_{0}\left(\sum_{k=1}^{n} a_{k} r_{k}\right)\right|=\left|\sum_{k=1}^{n} a_{k}\right| \leq C\left\|\sum_{k=1}^{n} a_{k} r_{k}\right\|_{K_{p}} .
$$

Therefore, $\varphi_{0}$ can be extended to a functional $\tilde{\varphi_{0}} \in\left(K_{p}\right)^{*}$. Since $\tilde{\varphi}_{0}\left(r_{n}\right)=$ $\varphi_{0}\left(r_{n}\right)=1$ for all $n \in \mathbb{N}$, it follows that $r_{n} \nrightarrow 0$ weakly in $K_{p}$.

Now, we show that the sequence

$$
s_{n}:=r_{n}-r_{n-1}, \quad n=1,2, \ldots, \quad \text { where } r_{0}=0,
$$

converges weakly to zero in $\mathcal{R}_{p}$, and it even forms a shrinking basis.

Proposition 1. The sequence $\left\{s_{n}\right\}_{n=1}^{\infty}$ is a shrinking basis in the space $\mathcal{R}_{p}$ for every $1 \leq p<\infty$. 
Proof. First, we show that $\left\{s_{n}\right\}_{n=1}^{\infty}$ is a basis in $\mathcal{R}_{p}$. To this end, we consider a function $f=\sum_{n=1}^{\infty} \beta_{n} s_{n} \in \mathcal{R}_{p}$. Since

$$
f=\sum_{n=1}^{\infty} \beta_{n}\left(r_{n}-r_{n-1}\right)=\sum_{n=0}^{\infty}\left(\beta_{n}-\beta_{n-1}\right) r_{n}, \quad \text { where } \beta_{0}=0,
$$

from 1.1 it follows that $f \in \mathcal{R}_{p}$ if and only if $\left\{\beta_{n}\right\}_{n=1}^{\infty}$ converges and $\sum_{n=1}^{\infty}\left(\beta_{n}-\beta_{n-1}\right)^{2}<\infty$. Moreover, $\left\{s_{n}\right\}_{n=1}^{\infty}$ is complete in $\mathcal{R}_{p}$ and

$$
\|f\|_{K_{p}} \asymp \sup _{n=1,2, \ldots}\left|\beta_{n}\right|+\left(\sum_{n=1}^{\infty}\left(\beta_{n}-\beta_{n-1}\right)^{2}\right)^{1 / 2},
$$

which implies that $\left\{s_{n}\right\}_{n=1}^{\infty}$ forms a basis in $\mathcal{R}_{p}$.

To prove the shrinking property of $\left\{s_{n}\right\}_{n=1}^{\infty}$ we need to show that for any $\varphi \in\left(K_{p}\right)^{*}$,

$$
\left\|\left.\varphi\right|_{\left[s_{n}\right]_{n=m}^{\infty}}\right\|_{K_{p}} \rightarrow 0 \quad \text { as } m \rightarrow \infty .
$$

Assume (2.3) does not hold. Then there exist $\varepsilon \in(0,1)$, a functional $\varphi \in\left(K_{p}\right)^{*}$ with $\|\varphi\|_{\left(K_{p}\right)^{*}}=1$, and a sequence of functions $f_{n}=\sum_{k=m_{n}}^{\infty} a_{k}^{m_{n}} s_{k}$, where $m_{1}<m_{2}<\cdots$, such that $\left\|f_{n}\right\|_{K_{p}}=1, n=1,2, \ldots$, and

$$
\varphi\left(f_{n}\right) \geq \varepsilon \quad \text { for all } n=1,2, \ldots
$$

It is not hard to construct two sequences of positive integers, $\left\{q_{i}\right\}_{i=1}^{\infty}$ and $\left\{p_{i}\right\}_{i=1}^{\infty}$, such that $q_{i}=m_{n_{i}}, i=1,2, \ldots, 1<q_{1}<p_{1}<q_{2}<p_{2}<\cdots$ and

$$
\left\|\sum_{n=p_{i}+1}^{\infty} a_{k}^{q_{i}} s_{k}\right\|_{K_{p}} \leq \frac{\varepsilon}{2} \text {. }
$$

In fact, setting $q_{1}=m_{1}$, we can find $p_{1}>q_{1}$ such that $\left\|\sum_{n=p_{1}+1}^{\infty} a_{k}^{q_{1}} s_{k}\right\|_{K_{p}} \leq$ $\varepsilon / 2$. Then, taking for $q_{2}$ the smallest $m_{n}$ which is larger than $p_{1}$, we find $p_{2}>q_{2}$ satisfying $\left\|\sum_{n=p_{2}+1}^{\infty} a_{k}^{q_{2}} s_{k}\right\|_{K_{p}} \leq \varepsilon / 2$. Continuing in the same way, we come to the required sequences $\left\{q_{i}\right\}_{i=1}^{\infty}$ and $\left\{p_{i}\right\}_{i=1}^{\infty}$.

Since $\left\|f_{n}\right\|_{K_{p}}=1, n=1,2, \ldots$, by (2.5) the sequence

$$
u_{i}:=\sum_{k=q_{i}}^{p_{i}} a_{k}^{q_{i}} s_{k}, \quad i=1,2, \ldots,
$$

is bounded in $K_{p}$. Moreover, from (2.4) and 2.5) it follows that

$$
\begin{aligned}
\varphi\left(u_{i}\right) & =\varphi\left(f_{i}\right)-\varphi\left(\sum_{k=p_{i}+1}^{\infty} a_{k}^{q_{i}} s_{k}\right) \\
& \geq \varphi\left(f_{i}\right)-\left\|\sum_{k=p_{i}+1}^{\infty} a_{k}^{q_{i}} s_{k}\right\|_{K_{p}} \geq \frac{\varepsilon}{2}, \quad i=1,2, \ldots
\end{aligned}
$$


Setting $\alpha_{k}^{i}=a_{k}^{q_{i}}$ if $q_{i} \leq k \leq p_{i}$ and $\alpha_{k}^{i}=0$ if $p_{i}<k<q_{i+1}, i=1,2, \ldots$, we have

$$
u_{i}:=\sum_{k=q_{i}}^{q_{i+1}-1} \alpha_{k}^{i} s_{k}, \quad i=1,2, \ldots
$$

that is, $\left\{u_{i}\right\}_{i=1}^{\infty}$ is a bounded block basis of $\left\{s_{k}\right\}_{k=1}^{\infty}$.

Let $\left\{\gamma_{n}\right\}_{n=1}^{\infty}$ be an arbitrary sequence of positive numbers such that

$$
\sum_{n=1}^{\infty} \gamma_{n}^{2}<\infty \text { and } \sum_{n=1}^{\infty} \gamma_{n}=\infty
$$

We want to show that the series $\sum_{n=1}^{\infty} \gamma_{n} u_{n}$ converges in $K_{p}$. To this end, we set

$$
b_{k}:=\alpha_{k}^{i} \gamma_{i} \quad \text { if } q_{i} \leq k<q_{i+1}, i=1,2, \ldots
$$

Then, by 2.2), if $k \geq q_{j}$ with some $j=1,2, \ldots$, we have

$$
\begin{aligned}
\left|b_{k}\right| & \leq \sup _{i \geq j} \max _{q_{i} \leq k<q_{i+1}}\left|a_{k}^{q_{i}} \gamma_{i}\right| \leq C \sup _{i \in \mathbb{N}}\left\|u_{i}\right\|_{K_{p}} \sup _{i \geq j} \gamma_{i} \\
& \leq C \sup _{i \in \mathbb{N}}\left\|u_{i}\right\|_{K_{p}} \sup _{i \geq j} \gamma_{i} .
\end{aligned}
$$

Hence, thanks to (2.7), we obtain $\lim _{k \rightarrow \infty} b_{k}=0$. Moreover,

$$
\begin{aligned}
\sum_{k=1}^{\infty}\left(b_{k}-b_{k+1}\right)^{2}= & \sum_{i=1}^{\infty} \sum_{k=q_{i}}^{q_{i+1}-2}\left(\alpha_{k}^{i} \gamma_{i}-\alpha_{k+1}^{i} \gamma_{i}\right)^{2} \\
& +\sum_{i=1}^{\infty}\left(\alpha_{q_{i+1}-1}^{i} \gamma_{i}-\alpha_{q_{i+1}}^{i+1} \gamma_{i+1}\right)^{2}=A_{1}+A_{2}
\end{aligned}
$$

Let us estimate $A_{1}$ and $A_{2}$ separately. In view of $(2.2)$ and $(2.7)$ we have

$$
A_{1}=\sum_{i=1}^{\infty} \gamma_{i}^{2} \sum_{k=q_{i}}^{q_{i+1}-2}\left(\alpha_{k}^{i}-\alpha_{k+1}^{i}\right)^{2} \leq C \sup _{i \in \mathbb{N}}\left\|u_{i}\right\|_{K_{p}} \sum_{i=1}^{\infty} \gamma_{i}^{2}<\infty,
$$

and similarly

$$
\left.A_{2} \leq 2 \sum_{i=1}^{\infty}\left[\left(\alpha_{q_{i+1}-1}^{i}\right)^{2} \gamma_{i}^{2}+\left(\alpha_{q_{i+1}}^{i+1}\right)^{2} \gamma_{i+1}\right)^{2}\right] \leq C \sum_{i=1}^{\infty} \gamma_{i}^{2} \sup _{i \in \mathbb{N}}\left\|u_{i}\right\|_{K_{p}}<\infty .
$$

The above observations combined with $(2.2)$ show that the series

$$
\sum_{n=1}^{\infty} \gamma_{n} u_{n}=\sum_{k=1}^{\infty} b_{k} s_{k}
$$

converges in $K_{p}$. At the same time, since $\varphi \in\left(K_{p}\right)^{*}$, by (2.6) and (2.7) we have

$$
\varphi\left(\sum_{n=1}^{\infty} \gamma_{n} u_{n}\right)=\sum_{n=1}^{\infty} \gamma_{n} \varphi\left(u_{n}\right) \geq \frac{\varepsilon}{2} \sum_{n=1}^{\infty} \gamma_{n}=\infty
$$

and therefore 2.3 is proved. 
Corollary 1. Let $\left\{u_{n}\right\}_{n=1}^{\infty}$ be a block basis defined as in (2.1), suppose $\left\|u_{n}\right\|_{K_{p}} \leq C, n=1,2, \ldots$, for some $C>0$, and let

$$
\gamma_{n}=\gamma_{n}\left(\left\{u_{n}\right\}\right):=\sum_{k=m_{n}+1}^{m_{n+1}} a_{k}, \quad n=1,2, \ldots
$$

Then $u_{n} \rightarrow 0$ weakly in $K_{p}$ if and only if $\gamma_{n} \rightarrow 0$ as $n \rightarrow \infty$.

Proof. Denote by $\left\{s_{n}^{*}\right\}_{n=1}^{\infty}$ the system biorthogonal to the above basis $\left\{s_{n}\right\}_{n=1}^{\infty}$. From Proposition 1 and [12, Proposition 1.b.1] it follows that $\left\{s_{n}^{*}\right\}_{n=1}^{\infty}$ is a basis in the dual space $\left(\mathcal{R}_{p}\right)^{*}$. By definition, we have

$$
s_{n}^{*}\left(s_{m}\right)=0 \text { if } n \neq m \text { and } s_{n}^{*}\left(s_{n}\right)=1, n=1,2, \ldots
$$

Since $s_{1}=r_{1}$, this implies that

$$
s_{n}^{*}\left(r_{m}\right)=1 \text { if } n \leq m \text { and } s_{n}^{*}\left(r_{m}\right)=0 \text { if } n>m .
$$

Now, define the sequence $\left\{r_{n}^{*}\right\}_{n=0}^{\infty}$ from $\left(\mathcal{R}_{p}\right)^{*}$ by setting

$$
r_{0}^{*}=s_{1}^{*} \quad \text { and } \quad r_{n}^{*}=s_{n}^{*}-s_{n+1}^{*}, \quad n=1,2, \ldots
$$

Clearly, $\left\{r_{n}^{*}\right\}_{n=0}^{\infty}$ is complete in $\left(\mathcal{R}_{p}\right)^{*}$ together with $\left\{s_{n}^{*}\right\}_{n=1}^{\infty}$, and from 2.8 it follows that

$r_{0}^{*}\left(r_{m}\right)=1(m=1,2, \ldots), \quad r_{n}^{*}\left(r_{m}\right)=0(n \neq m), \quad r_{n}^{*}\left(r_{n}\right)=1(n=1,2, \ldots)$.

Since $r_{0}^{*}\left(u_{n}\right)=\gamma_{n}, n=1,2, \ldots$, we have

$$
r_{k}^{*}\left(u_{n}\right) \rightarrow 0 \quad \text { for every } k=0,1,2, \ldots
$$

if and only if $\gamma_{n} \rightarrow 0$ as $n \rightarrow \infty$. On the other hand, in view of the boundedness of $\left\{u_{n}\right\}_{n=1}^{\infty}$ in $K_{p}$, condition 2.9 is equivalent to the weak convergence of $\left\{u_{n}\right\}$ to zero in $K_{p}$. Therefore, the result follows.

Proof of Theorem 3. Let $\left\{u_{n}\right\}_{n=1}^{\infty}$ be a block basis of the Rademacher functions defined in 2.1. First, by assumption and Corollary 1, we have

$$
\gamma_{n}:=\sum_{k=m_{n}+1}^{m_{n+1}} a_{k} \rightarrow 0 \quad \text { as } n \rightarrow \infty,
$$

and passing to a subsequence of $\left\{u_{n}\right\}_{n=1}^{\infty}$ if necessary, we can assume that

$$
\left|\gamma_{n}\right| \leq 2^{-n}, \quad n=1,2, \ldots
$$

(a) Let $f=\sum_{n=1}^{\infty} b_{n} u_{n} \in \mathcal{R}_{p}$. Then

$$
f=\sum_{n=1}^{\infty} \sum_{k=m_{n}+1}^{m_{n+1}} b_{n} a_{k} r_{k}=\sum_{k=1}^{\infty} \beta_{k} r_{k} .
$$

First, we estimate $\sum_{k=p}^{q} \beta_{k}$ for $p \leq q$. Let $m_{n-1} \leq p<m_{n}<m_{n+l}<q \leq$ 
$m_{n+l+1}$ for some $n$ and $l$. Then

$$
\begin{aligned}
\left|\sum_{k=p}^{q} \beta_{k}\right| & =\left|\sum_{k=p}^{m_{n}} \beta_{k}+\sum_{k=m_{n}+1}^{m_{n+l}} \beta_{k}+\sum_{k=m_{n+l}+1}^{q} \beta_{k}\right| \\
& =\left|\sum_{k=p}^{m_{n}} b_{n-1} a_{k}+\sum_{i=n}^{n+l-1} \sum_{k=m_{i}+1}^{m_{i+1}} b_{i} a_{k}+\sum_{k=m_{n+l}+1}^{q} b_{n+l} a_{k}\right| \\
& \leq\left|b_{n-1}\right|\left|\sum_{k=p}^{m_{n}} a_{k}\right|+\sum_{i=n}^{n+l-1}\left|b_{i}\right|\left|\sum_{k=m_{i}+1}^{m_{i+1}} a_{k}\right|+\left|b_{n+l}\right|\left|\sum_{k=m_{n+l}+1}^{q} a_{k}\right| \\
& \leq \sup _{n \in \mathbb{N}}\left|b_{n}\right|\left(\left|\sum_{k=p}^{m_{n}} a_{k}\right|+\sum_{i=n}^{n+l-1}\left|\sum_{k=m_{i}+1}^{m_{i+1}} a_{k}\right|+\left|\sum_{k=m_{n+l}+1}^{q} a_{k}\right|\right) .
\end{aligned}
$$

By 1.1, we have

$$
\max \left(\left|\sum_{k=p}^{m_{n}} a_{k}\right|,\left|\sum_{k=m_{n+l}+1}^{q} a_{k}\right|\right) \leq C\left\|u_{n}\right\|_{K_{p}} \leq C_{1} .
$$

Moreover, from 2.10 it follows that

$$
\sum_{i=n}^{n+l-1}\left|\sum_{k=m_{i}+1}^{m_{i+1}} a_{k}\right|=\sum_{i=n}^{n+l-1}\left|\gamma_{i}\right| \leq \sum_{i=n}^{n+l-1} 2^{-i}<1 .
$$

Therefore, from the preceding estimates we infer that

$$
\begin{aligned}
\left|\sum_{k=p}^{q} \beta_{k}\right| & \leq\left(2 C_{1}+1\right) \sup _{n \in \mathbb{N}}\left|b_{n}\right| \\
& \leq\left(2 C_{1}+1\right)\left(\sum_{n=1}^{\infty} b_{n}^{2}\right)^{1 / 2} \quad \text { for all } 1 \leq p \leq q<\infty .
\end{aligned}
$$

By assumption and (1.1), there is a constant $C_{2}>0$ such that for all $n \in \mathbb{N}$,

$$
\sum_{k=m_{n}+1}^{m_{n+1}} a_{k}^{2} \leq C\left\|u_{n}\right\|_{K_{p}}^{2} \leq C_{2}^{2}
$$

and so

$$
\left(\sum_{k=1}^{\infty} \beta_{k}^{2}\right)^{1 / 2} \leq\left(\sum_{n=1}^{\infty} b_{n}^{2} \cdot \sum_{k=m_{n}+1}^{m_{n+1}} a_{k}^{2}\right)^{1 / 2} \leq C_{2}\left(\sum_{n=1}^{\infty} b_{n}^{2}\right)^{1 / 2}
$$

On the other hand, since $\left\|u_{n}\right\|_{d}=\left(\sum_{k=m_{n}+1}^{m_{n+1}} a_{k}^{2}\right)^{1 / 2} \geq \varepsilon$, we have

$$
\left(\sum_{k=1}^{\infty} \beta_{k}^{2}\right)^{1 / 2}=\left(\sum_{n=1}^{\infty} b_{n}^{2}\left\|u_{n}\right\|_{d}^{2}\right)^{1 / 2} \geq \varepsilon\left(\sum_{n=1}^{\infty} b_{n}^{2}\right)^{1 / 2} .
$$


From 2.12 -2.14 and (1.1), it follows that $\left\{u_{n}\right\}_{n=1}^{\infty}$ is equivalent to the unit vector basis in $l_{2}$, so the proof of part (a) is complete.

(b) Since $\left\|u_{n}\right\|_{d} \rightarrow 0$ as $n \rightarrow \infty$, we can assume that

$$
\left\|u_{n}\right\|_{d} \leq \eta 2^{-n}, \quad n=1,2, \ldots,
$$

where $\eta>0$ will be chosen later on. Suppose that $f=\sum_{n=1}^{\infty} b_{n} u_{n} \in \mathcal{R}_{p}$. Then, representing $f$ by formula (2.11), and applying (1.1), (2.15) and the first inequality of (2.12) (which is still valid), we obtain

$$
\begin{aligned}
\|f\|_{K_{p}} & \leq C\left(\|f\|_{d}+\sup _{1 \leq p \leq q<\infty}\left|\sum_{k=p}^{q} \beta_{k}\right|\right) \\
& \leq C\left(\sum_{n=1}^{\infty}\left|b_{n}\right|\left\|u_{n}\right\|_{d}+\left(2 C_{1}+1\right) \sup _{n \in \mathbb{N}}\left|b_{n}\right|\right) \leq C_{3} \sup _{n \in \mathbb{N}}\left|b_{n}\right| .
\end{aligned}
$$

On the other hand, by (1.1), we have

$$
\|f\|_{K_{p}} \geq c \sup _{1 \leq p \leq q<\infty}\left|\sum_{k=p}^{q} \beta_{k}\right| \geq c \sup _{n \in \mathbb{N}}\left|b_{n}\right| \cdot \max _{m_{n}<j \leq m_{n+1}}\left|\sum_{k=m_{n}+1}^{j} a_{k}\right| .
$$

Since $\left\|u_{n}\right\|_{K_{p}} \geq 1 / c_{0}, n=1,2, \ldots$, choosing $\eta>0$ in 2.15) sufficiently small, for every $n \in \mathbb{N}$ we can find $l_{n}$ with $m_{n}<l_{n} \leq m_{n+1}$ such that for some $\delta>0$,

$$
\left|\sum_{k=m_{n}+1}^{l_{n}} a_{k}\right| \geq \delta
$$

Combining this observation with the preceding estimate we obtain

$$
\|f\|_{K_{p}} \geq c \delta \sup _{n \in \mathbb{N}}\left|b_{n}\right|,
$$

and therefore (b) is proved.

Now, we are ready to prove the main result of Section 2 .

TheOREm 4. Let $1 \leq p<\infty$. Every infinite-dimensional subspace $X$ of $\mathcal{R}_{p}$ either is isomorphic to $l_{2}$, or contains a subspace isomorphic to $c_{0}$ and complemented in $\mathcal{R}_{p}$.

Proof. Suppose that for every $f=\sum_{k=1}^{\infty} b_{k} r_{k} \in X$ we have

$$
\|f\|_{K_{p}} \asymp\|f\|_{d}=\left(\sum_{k=1}^{\infty} b_{k}^{2}\right)^{1 / 2} .
$$

This means that $X$ is isomorphic to some subspace of $l_{2}$ and so to $l_{2}$ itself.

Otherwise, by (1.1), there is a sequence $\left\{f_{n}\right\}_{n=1}^{\infty} \subset X$ with $\left\|f_{n}\right\|_{K_{p}}=1$ for which $\left\|f_{n}\right\|_{d} \rightarrow 0$ as $n \rightarrow \infty$. Observe that $\left\{f_{n}\right\}_{n=1}^{\infty}$ has no subsequence converging in $K_{p}$-norm. In fact, if $\left\|f_{n_{k}}-f\right\|_{K_{p}} \rightarrow 0$ for some $\left\{f_{n_{k}}\right\} \subset\left\{f_{n}\right\}$ and $f \in X$, then $\left\|f_{n_{k}}-f\right\|_{d} \rightarrow 0$ and hence $\|f\|_{d}=0$, i.e., $f=0$. On the 
other hand, $\|f\|_{K_{p}}$ should be equal to 1 , and we come to a contradiction. Thus, passing to a subsequence if necessary, we can assume that

$$
\left\|f_{n}-f_{m}\right\|_{K_{p}} \geq \varepsilon>0 \quad \text { for all } n \neq m .
$$

Recall that $\left\{s_{n}^{*}\right\}_{n=1}^{\infty}$ is a basis in $\left(\mathcal{R}_{p}\right)^{*}$. Applying the diagonal process, we can construct a sequence $\left\{n_{k}\right\}_{k=1}^{\infty}, n_{1}<n_{2}<\cdots$, such that $\lim _{k \rightarrow \infty} s_{i}^{*}\left(f_{n_{k}}\right)$ exists for every $i=1,2, \ldots$. Then

$$
\lim _{k \rightarrow \infty} s_{i}^{*}\left(f_{n_{2 k+1}}-f_{n_{2 k}}\right)=0 \quad \text { for all } i=1,2, \ldots,
$$

and since $\left\{f_{n_{2 k+1}}-f_{n_{2 k}}\right\}_{k=1}^{\infty}$ is bounded in $\mathcal{R}_{p}$, we infer that $f_{n_{2 k+1}}-f_{n_{2 k}} \rightarrow 0$ weakly in $K_{p}$. Now, taking into account $(2.16)$ and applying the well-known Bessaga-Pełczyński Selection Principle (see [1, Proposition 1.3.10] or [12, Proposition 1.a.12]), we can find a subsequence of $\left\{f_{n_{2 k+1}}-f_{n_{2 k}}\right\}$ (not relabelled) and a block basis $\left\{u_{k}\right\}_{k=1}^{\infty}$ of the Rademacher functions such that

$$
\left\|u_{k}-\left(f_{n_{2 k+1}}-f_{n_{2 k}}\right)\right\|_{K_{p}} \leq B_{0}^{-1} 2^{-k-1}, \quad k=1,2, \ldots,
$$

where $B_{0}$ is the basic constant of $\left\{r_{k}\right\}$ in $\mathcal{R}_{p}$. Then the sequences $\left\{u_{k}\right\}_{k=1}^{\infty}$ and $\left\{f_{n_{2 k+1}}-f_{n_{2 k}}\right\}_{k=1}^{\infty}$ are equivalent in $K_{p}$ (cf. [12, Proposition 1.a.9]). Moreover, from (2.17) it follows that $u_{k} \rightarrow 0$ weakly in $K_{p}$ and $\left\|u_{k}\right\|_{d} \rightarrow 0$. Therefore, by Theorem 3 (b), the sequence $\left\{u_{k}\right\}_{k=1}^{\infty}$ (and so $\left\{f_{n_{2 k+1}}-f_{n_{2 k}}\right\}_{k=1}^{\infty}$ ) contains a subsequence equivalent to the unit vector basis of $c_{0}$. Complementability in $\mathcal{R}_{p}$ of the subspace spanned by the latter subsequence is an immediate consequence of Sobczyk's theorem [1, Corollary 2.5.9].

Arguing as in the proof of Theorem 4 , we also obtain the following result.

TheOREM 5. Let $1 \leq p<\infty$, and let $\left\{f_{n}\right\}_{n=1}^{\infty}$ be a basic sequence in $\mathcal{R}_{p}$ weakly converging to zero in $K_{p}$ with $1 / c_{0} \leq\left\|f_{n}\right\|_{K_{p}} \leq c_{0}$ for some constant $c_{0}>0$ and for all $n \in \mathbb{N}$. Then $\left\{f_{n}\right\}_{n=1}^{\infty}$ contains a subsequence equivalent to the unit vector basis of $l_{2}$ or $c_{0}$.

3. Structure of Rademacher subspaces in $K$. In this section we complete the proof of Theorem 1 1 . In view of Theorem 3 all we need is the following result.

TheOREM 6. Let $X$ be a subspace of $K=\mathrm{Ces}_{\infty}$ which is isomorphic to $l_{2}$ and such that $X \subset \mathcal{R}$. Then $X$ is uncomplemented in $K$.

Proof. On the contrary, assume that an $X$ as above is complemented in $K$. Let $\left\{x_{n}\right\}_{n=1}^{\infty} \subset X$ be equivalent to the unit vector basis $\left\{e_{n}\right\}_{n=1}^{\infty}$ of $l_{2}$. Since $e_{n} \stackrel{w}{\rightarrow} 0$ in $l_{2}$, it follows that $x_{n} \stackrel{w}{\rightarrow} 0$ in $K$. Noting that $x_{n} \in \mathcal{R}$ and $\left\|x_{n}\right\|_{K} \asymp 1, n=1,2, \ldots$, by applying the Bessaga-Pełczyński theorem once more (see [1, Proposition 1.3.10] or [12, Proposition 1.a.12]), we select 
a subsequence $\left\{x_{n_{i}}\right\} \subset\left\{x_{n}\right\}$ equivalent to a suitable block basis $\left\{u_{n}\right\}$ of the Rademacher functions,

$$
u_{n}=\sum_{k=m_{n}+1}^{m_{n+1}} a_{k} r_{k}, \quad 0<m_{1}<m_{2}<\cdots, n \in \mathbb{N},
$$

such that $\left\|x_{n_{i}}-u_{i}\right\|_{K} \rightarrow 0$ as $i \rightarrow \infty$. Hence, $\left\{u_{n}\right\}$ is equivalent in $K$ to the unit vector basis in $l_{2}$, i.e.,

$$
\left\|\sum_{n=1}^{\infty} b_{n} u_{n}\right\|_{K} \asymp\left(\sum_{n=1}^{\infty} b_{n}^{2}\right)^{1 / 2}, \quad b_{n} \in \mathbb{R} .
$$

Taking into account the principle of small perturbations [1, Proposition 1.3.9], we can also assume that the closed linear span $\left[u_{n}\right]$ is also complemented in $K$. Moreover,

$$
\left\|u_{n}\right\|_{K} \asymp\left(\sum_{k=m_{n}+1}^{m_{n+1}} a_{k}^{2}\right)^{1 / 2} \asymp 1, \quad n=1,2, \ldots .
$$

In fact, otherwise we have

$$
\liminf _{n \rightarrow \infty} \sum_{k=m_{n}+1}^{m_{n+1}} a_{k}^{2}=0 .
$$

Also if $\varphi \in K^{*}$, then

$$
\left|\varphi\left(u_{i}\right)\right| \leq\left|\varphi\left(u_{i}-x_{n_{i}}\right)\right|+\left|\varphi\left(x_{n_{i}}\right)\right| \leq\|\varphi\|\left\|u_{i}-x_{n_{i}}\right\|+\left|\varphi\left(x_{n_{i}}\right)\right| .
$$

Since $x_{n} \stackrel{w}{\rightarrow} 0$ in $K$ and $\left\|u_{i}-x_{n_{i}}\right\|_{K} \rightarrow 0$, we find that $u_{n} \stackrel{w}{\rightarrow} 0$ in $K$ as well. Therefore, by Theorem 3 (b), $\left\{u_{n}\right\}_{n=1}^{\infty}$ contains a subsequence $\left\{u_{n_{i}}\right\}_{i=1}^{\infty}$ equivalent in $K$ to the unit vector basis in $c_{0}$. Since this contradicts $\left\{u_{n}\right\}$ being equivalent in $K$ to the unit vector basis in $l_{2},(3.2)$ is proved.

Let $P$ be a bounded projection from $K$ onto $\left[u_{n}\right]$. Since $\left\{u_{n}\right\}$ is a basic sequence in $K$, we can find functionals $\varphi_{n} \in K^{*}, n=1,2, \ldots$, such that

$$
\operatorname{Pf}(x)=\sum_{n=1}^{\infty} \varphi_{n}(f) u_{n}(x), \quad f \in K .
$$

Since $K \subset L_{1}$, the Köthe dual $K^{\prime}$ contains the space $L_{\infty}$, and therefore $K^{\prime}$ is a total set on $K$. Thus, by [9, Chapter 10, Theorem 3.6], we have

$$
K^{*}=K^{c} \oplus K^{s},
$$

where $K^{c}$ (respectively, $K^{s}$ ) is the set of all order continuous linear functionals on $K$ generated by the space $K^{\prime}$ (respectively, singular bounded linear functionals on $K$ ). Hence, if $\theta \in K^{s}$, then

$$
\theta(f)=0 \quad \text { for every } f \in K_{0},
$$


where $K_{0}$ is the separable part of $K$ (the set of all elements in $K$ having absolutely continuous norm). In particular, from (3.3) it follows that

$$
\varphi_{n}=\psi_{n}+\theta_{n} \quad \text { with } \psi_{n} \in K^{c} \text { and } \theta_{n} \in K^{s}, n=1,2, \ldots
$$

Moreover, since $P$ is a projection onto $\left[u_{n}\right]$, we have

$$
\psi_{n}\left(u_{n}\right)+\theta_{n}\left(u_{n}\right)=1(n=1,2, \ldots), \quad \psi_{n}\left(u_{i}\right)+\theta_{n}\left(u_{i}\right)=0 \text { for } i \neq n .
$$

Since from (3.1) it follows that

$$
\|P f\|_{K} \asymp\left(\sum_{n=1}^{\infty} \varphi_{n}(f)^{2}\right)^{1 / 2}<\infty \quad \text { for every } f \in K,
$$

$\left\{\varphi_{n}\right\}$ is a weakly* null sequence in $K^{*}$. Therefore,

$$
\left\|\varphi_{n}\right\|_{K^{*}} \leq A \quad(n=1,2, \ldots) \text { for some } A>0 .
$$

On the other hand, taking into account (3.4), we see that the operator

$$
Q f(x)=\sum_{n=1}^{\infty} \psi_{n}(f) u_{n}(x), \quad f \in K_{0},
$$

coincides with $P$ on $K_{0}$ and hence $Q: K_{0} \rightarrow K$ is bounded. Let us show that $Q$ acts boundedly in $K$.

Since $\psi_{n} \in K^{c}$, we have

$$
\psi_{n}(f)=\int_{0}^{1} g_{n}(t) f(t) d t \quad \text { for some } g_{n} \in K^{\prime} .
$$

For every $f \in K$ we have $|f| \chi_{[1 / m, 1]} \cdot \operatorname{sign} g_{n} \in K_{0}, m, n=1,2, \ldots$, and therefore in view of (3.6) and (3.4),

$$
\begin{aligned}
\int_{0}^{1}\left|g_{n}(t) f(t) \chi_{[1 / m, 1]}(t)\right| d t & =\psi_{n}\left(|f| \chi_{[1 / m, 1]} \cdot \operatorname{sign} g_{n}\right) \\
& =\varphi_{n}\left(|f| \chi_{[1 / m, 1]} \operatorname{sign} g_{n}\right) \\
& \leq A\|f\|_{K}, \quad m, n=1,2, \ldots
\end{aligned}
$$

Letting $m \rightarrow \infty$, by the Fatou lemma we have

$$
\int_{0}^{1}\left|g_{n}(t) f(t)\right| d t \leq A\|f\|_{K} \quad \text { for all } f \in K \text { and } n \in \mathbb{N},
$$

whence $\left\|\psi_{n}\right\|_{K^{*}} \leq A$. Combining this inequality with (3.6), we infer that

$$
\left\|\theta_{n}\right\|_{K^{*}} \leq 2 A, \quad n=1,2, \ldots
$$


Moreover, by (3.1),

$$
\begin{aligned}
\sum_{n=1}^{\infty}\left(\int_{0}^{1} g_{n}(t) f(t) \chi_{[1 / m, 1]}(t) d t\right)^{2} & \asymp\left\|Q\left(f \chi_{[1 / m, 1]}\right)\right\|_{K}^{2} \\
& \leq C\left\|f \chi_{[1 / m, 1]}\right\|_{K}^{2} \leq C\|f\|_{K}^{2} .
\end{aligned}
$$

Since $g_{n} f \in L_{1}$ for all $n=1,2, \ldots$, as above we obtain

$$
\|Q f\|_{K}^{2} \asymp \sum_{n=1}^{\infty}\left(\int_{0}^{1} g_{n}(t) f(t) d t\right)^{2} \leq C\|f\|_{K}^{2},
$$

and the assertion is proved.

Note that $r_{i}-\chi_{[0,1]} \in K_{0}$ for all $i \in \mathbb{N}$. Therefore, by (3.5), $\theta_{n}\left(r_{i}\right)=$ $\theta_{n}\left(\chi_{[0,1]}\right):=c_{n}$ for all $n, i \in \mathbb{N}$, and

$$
\theta_{n}\left(u_{i}\right)=c_{n} \sum_{k=m_{i}+1}^{m_{i+1}} a_{k}, \quad n, i \in \mathbb{N} .
$$

Moreover, since $u_{i} \stackrel{w}{\rightarrow} 0$ in $K$, by Corollary 1 we obtain

$$
\sum_{k=m_{i}+1}^{m_{i+1}} a_{k} \rightarrow 0 \quad \text { as } i \rightarrow \infty .
$$

Therefore, by (3.8), for all positive integers $n$ and $i$,

$$
\left|c_{n}\right|=\left|\theta_{n}\left(\chi_{[0,1]}\right)\right| \leq\left\|\theta_{n}\right\|_{K^{*}}\left\|\chi_{[0,1]}\right\|_{K} \leq 2 A,
$$

whence

$$
\lim _{i \rightarrow \infty} \sup _{n \in \mathbb{N}}\left|\theta_{n}\left(u_{i}\right)\right|=0 .
$$

On the other hand, by (3.5), we have

$$
\psi_{n}\left(u_{i}\right)=-\theta_{n}\left(u_{i}\right)(i \neq n) \text { and } \psi_{n}\left(u_{n}\right)=1-\theta_{n}\left(u_{n}\right), \quad n \in \mathbb{N},
$$

whence

$$
\lim _{n \rightarrow \infty} \psi_{n}\left(u_{n}\right)=1 \quad \text { and } \quad \lim _{i \rightarrow \infty} \sup _{n \neq i}\left|\psi_{n}\left(u_{i}\right)\right|=0 .
$$

Thus, passing to subsequences of $\left\{u_{n}\right\}$ and $\left\{\psi_{n}\right\}$, and keeping the same notation, we deduce that the operator

$$
R f(x):=\sum_{n=1}^{\infty} \psi_{n}(f) u_{n}(x), \quad f \in K,
$$

where

$$
\psi_{n}\left(u_{n}\right) \geq 1-2^{-n}(n=1,2, \ldots) \quad \text { and } \quad\left|\psi_{n}\left(u_{i}\right)\right| \leq 2^{-i}, n \neq i,
$$

acts boundedly in $K$. As above, the functionals $\psi_{n}$ are defined by (3.7).

Since $K \subset L_{1}[0,1]$, the operator $R$ is also bounded from $K$ into $L_{1}$. Let us show that $R: K \rightarrow L_{1}$ is weakly compact. By the Dunford-Pettis 
theorem (see, for example, [1, Theorem 5.2.9]), it is sufficient to check that the set $\left\{R f:\|f\|_{K} \leq 1\right\}$ is uniformly integrable on $[0,1]$. In fact, by (3.2) and (3.9), for every $f \in K$ with $\|f\|_{K} \leq 1$ and any set $E \subset[0,1]$, we obtain

$$
\begin{aligned}
\left\|R f \cdot \chi_{E}\right\|_{L_{1}} & \leq m(E)^{1 / 2}\|R f\|_{L_{2}}=m(E)^{1 / 2}\left(\sum_{n=1}^{\infty} \psi_{n}(f)^{2} \cdot \sum_{k=m_{n}+1}^{m_{n+1}} a_{k}^{2}\right)^{1 / 2} \\
& \asymp m(E)^{1 / 2}\left(\sum_{n=1}^{\infty} \psi_{n}(f)^{2}\right)^{1 / 2} \leq C\|R\| m(E)^{1 / 2}
\end{aligned}
$$

whence

$$
\lim _{m(E) \rightarrow 0} \sup \left\{\left\|R f \cdot \chi_{E}\right\|_{L_{1}}:\|f\|_{K} \leq 1\right\}=0
$$

(here, $m(E)$ is the Lebesgue measure of a set $E$ ). Thus, $R$ is a weakly compact operator from $K$ into $L_{1}$.

Now, we consider separately two cases. Firstly, assume that there are $\delta \in(0,1)$ and a subsequence $\left\{u_{n_{k}}\right\} \subset\left\{u_{n}\right\}$ such that

$$
\left|\psi_{n_{k}}\left(u_{n_{k}} \cdot \chi_{[\delta, 1]}\right)\right|=\left|\int_{\delta}^{1} g_{n_{k}}(t) u_{n_{k}}(t) d t\right| \geq \frac{1}{2}, \quad k=1,2, \ldots
$$

Note that for every measurable function $f$ on $[0,1]$ with supp $f \subset[\delta, 1]$ we have

$$
\|f\|_{L_{1}[\delta, 1]} \leq\|f\|_{K}=\sup _{\delta \leq x \leq 1} \frac{1}{x} \int_{\delta}^{1}|f(t)| d t \leq \frac{1}{\delta}\|f\|_{L_{1}[\delta, 1]} .
$$

Therefore, $R_{\delta} f:=R\left(f \chi_{[\delta, 1]}\right)$ is a weakly compact operator in $L_{1}[0,1]$. Since $L_{1}[0,1]$ has the Dunford-Pettis property (see [1, Theorem 5.4.5]), we conclude that $R_{\delta}$ is weak-to-norm sequentially continuous. Clearly, from $u_{n} \stackrel{w}{\rightarrow} 0$ in $K$ it follows that $u_{n} \chi_{[\delta, 1]} \stackrel{w}{\rightarrow} 0$ in $L_{1}[0,1]$. Thus, $\left\|R\left(u_{n} \chi_{[\delta, 1]}\right)\right\|_{L_{1}} \rightarrow 0$ as $n \rightarrow \infty$. On the other hand, by the Khintchine inequality in $L_{1}, 3.2$ and (3.11), we have

$$
\begin{aligned}
\left\|R\left(u_{n_{k}} \cdot \chi_{[\delta, 1]}\right)\right\|_{L_{1}} & \asymp\left\|\sum_{i=1}^{\infty} \psi_{i}\left(u_{n_{k}} \cdot \chi_{[\delta, 1]}\right) \sum_{j=m_{i}+1}^{m_{i+1}} a_{j} r_{j}\right\|_{L_{1}} \\
& \asymp\left[\sum_{i=1}^{\infty} \psi_{i}\left(u_{n_{k}} \cdot \chi_{[\delta, 1]}\right)^{2} \sum_{j=m_{i}+1}^{m_{i+1}} a_{j}^{2}\right]^{1 / 2} \\
& \asymp\left[\sum_{i=1}^{\infty} \psi_{i}\left(u_{n_{k}} \cdot \chi_{[\delta, 1]}\right)^{2}\right]^{1 / 2} \geq\left|\psi_{n_{k}}\left(u_{n_{k}} \cdot \chi_{[\delta, 1]}\right)\right| \geq \frac{1}{2}
\end{aligned}
$$

for all $k=1,2, \ldots$ This contradiction concludes the proof in the case when (3.11) holds. 
Suppose now that (3.11) does not hold. Then, by (3.10) and (3.7), for any $\delta \in(0,1)$ and all sufficiently large $n \in \mathbb{N}$ we have

$$
\left|\int_{0}^{\delta} g_{n}(t) u_{n}(t) d t\right|>\frac{1}{4} .
$$

Setting $\delta_{0}=1 / 2$, we find $n_{1} \in \mathbb{N}$ and $\delta_{1} \in\left(0, \delta_{0}\right)$ such that

$$
\left|\int_{\delta_{1}}^{\delta_{0}} g_{n_{1}}(t) u_{n_{1}}(t) d t\right|>\frac{1}{4} \text {. }
$$

Denote $v_{n_{1}}:=u_{n_{1}} \chi_{\left(\delta_{1}, \delta_{0}\right)}$. From 3.9 it follows that $g_{n} \stackrel{w^{*}}{\rightarrow} 0$ in $K^{*}$. Moreover, we know that $u_{n} \stackrel{w}{\rightarrow} 0$ in $K$. Therefore, by 3.12 , there is $n_{2}>n_{1}$ for which

$$
\begin{aligned}
& \left|\int_{0}^{\delta_{1}} g_{n_{1}}(t) u_{n_{2}}(t) d t\right|<\frac{1}{2^{3}}, \\
& \left|\int_{0}^{1} g_{n_{2}}(t) v_{n_{1}}(t) d t\right|<\frac{1}{2^{3}}, \\
& \left|\int_{0}^{\delta_{1}} g_{n_{2}}(t) u_{n_{2}}(t) d t\right|>\frac{1}{4} .
\end{aligned}
$$

Furthermore, we can find $\delta_{2} \in\left(0, \delta_{1}\right)$ such that the functions $g_{n_{i}}$ and $v_{n_{i}}$ $(i=1,2)$, where $v_{n_{2}}:=u_{n_{2}} \chi_{\left[\delta_{2}, \delta_{1}\right]}$, satisfy

$$
\begin{aligned}
& \left|\int_{0}^{1} g_{n_{j}}(t) v_{n_{i}}(t) d t\right|<\frac{1}{2^{3}} \quad \text { for } 1 \leq i \neq j \leq 2, \\
& \left|\int_{0}^{1} g_{n_{2}}(t) v_{n_{2}}(t) d t\right|>\frac{1}{4} .
\end{aligned}
$$

Suppose that for some $k \in \mathbb{N}$ we have chosen

$$
n_{1}<\cdots<n_{k} \text { and } 1 / 2=\delta_{0}>\delta_{1}>\cdots>\delta_{k}>0
$$

so that the functions $g_{n_{i}}$ and $v_{n_{i}}:=u_{n_{i}} \chi_{\left[\delta_{i}, \delta_{i-1}\right]}, i=1, \ldots, k$, satisfy

$$
\begin{array}{ll}
\left|\int_{0}^{1} g_{n_{j}}(t) v_{n_{i}}(t) d t\right|<\frac{1}{2^{i+j}}, & 1 \leq i \neq j \leq k, \\
\left|\int_{0}^{1} g_{n_{i}}(t) v_{n_{i}}(t) d t\right|>\frac{1}{4}, & i=1, \ldots, k .
\end{array}
$$

Using the facts that $u_{n} \stackrel{w}{\rightarrow} 0$ in $K$ and $g_{n} \stackrel{w^{*}}{\rightarrow} 0$ in $K^{*}$, and 3.12 once more, 
we can find $n_{k+1}>n_{k}$ such that

$$
\begin{aligned}
\left|\int_{0}^{\delta_{k}} g_{n_{i}}(t) u_{n_{k+1}}(t) d t\right| & <\frac{1}{2^{i+k+1}}, \quad i=1, \ldots, k, \\
\left|\int_{0}^{1} g_{n_{k+1}}(t) v_{n_{i}}(t) d t\right| & <\frac{1}{2^{i+k+1}}, \quad i=1, \ldots, k, \\
\left|\int_{0}^{\delta_{k}} g_{n_{k+1}}(t) u_{n_{k+1}}(t) d t\right| & >\frac{1}{4} .
\end{aligned}
$$

Clearly, there is $\delta_{k+1} \in\left(0, \delta_{k}\right)$ such that for the functions $g_{n_{i}}$ and $v_{n_{i}}$, where $v_{n_{k+1}}:=u_{n_{k+1}} \chi_{\left[\delta_{k+1}, \delta_{k}\right]}$, inequalities (3.13) (respectively, (3.14) hold for all $1 \leq i \neq j \leq k+1$ (respectively, $i=1, \ldots, k+1$ ).

Thus, we can select sequences

$$
n_{1}<n_{2}<\cdots \quad \text { and } 1 / 2=\delta_{0}>\delta_{1}>\cdots>0
$$

such that the functions $g_{n_{i}}$ and $v_{n_{i}}:=u_{n_{i}} \chi_{\left[\delta_{i}, \delta_{i-1}\right]}, i=1,2, \ldots$, satisfy

$$
\begin{array}{ll}
\left|\int_{0}^{1} g_{n_{j}}(t) v_{n_{i}}(t) d t\right| \leq \frac{1}{2^{i+j}}, & 1 \leq i \neq j<\infty, \\
\left|\int_{0}^{1} g_{n_{i}}(t) v_{n_{i}}(t) d t\right|>\frac{1}{4}, & i=1,2, \ldots
\end{array}
$$

By [3, Proposition 1], every sequence $\left\{f_{n}\right\} \subset K$ such that supp $f_{n} \subset\left[a_{n}, b_{n}\right]$ with $b_{1}>a_{1}>b_{2}>a_{2}>\cdots>0$ and $b_{n} \rightarrow 0^{+}$contains a subsequence $\left\{f_{n_{k}}\right\}$ which is equivalent in $K$ to the unit vector basis of $c_{0}$. Therefore, we can assume that

$$
\sup _{m=1,2, \ldots}\left\|\sum_{i=1}^{m} v_{n_{i}}\right\|_{K} \leq C \sup _{i}\left\|v_{n_{i}}\right\|_{K} \leq C \sup _{n=1,2, \ldots}\left\|u_{n}\right\|_{K}<\infty .
$$

Moreover, it is clear that the operator

$$
R^{\prime} f(x):=\sum_{i=1}^{\infty} \int_{0}^{1} g_{n_{i}}(t) f(t) d t \cdot u_{n_{i}}(x)
$$

is bounded in $K$ together with the operator $R$. Hence, on the one hand,

$$
\left\|R^{\prime}\left(\sum_{i=1}^{m} v_{n_{i}}\right)\right\|_{K} \leq\left\|R^{\prime}\right\|\left\|\sum_{i=1}^{m} v_{n_{i}}\right\|_{K} \leq C\left\|R^{\prime}\right\| \quad \text { for all } m \in \mathbb{N} .
$$

On the other hand, by (3.1), (3.15) and (3.16), for every $m=1,2, \ldots$, we 
have

$$
\begin{aligned}
\left\|R^{\prime}\left(\sum_{i=1}^{m} v_{n_{i}}\right)\right\|_{K} & =\left\|\sum_{j=1}^{\infty} \sum_{i=1}^{m} \int_{0}^{1} g_{n_{j}}(s) v_{n_{i}}(s) d s \cdot u_{n_{j}}\right\|_{K} \\
& \geq\left\|\sum_{i=1}^{m} \int_{0}^{1} g_{n_{i}}(s) v_{n_{i}}(s) d s \cdot u_{n_{i}}\right\|_{K}-\sum_{i, j=1, i \neq j}^{\infty}\left|\int_{0}^{1} g_{n_{j}}(s) v_{n_{i}}(s) d s\right| \\
& \geq\left\|\sum_{i=1}^{m} \int_{0}^{1} g_{n_{i}}(s) v_{n_{i}}(s) d s \cdot u_{n_{i}}\right\|_{K}-1 \\
& \geq c\left[\sum_{i=1}^{m}\left|\int_{0}^{1} g_{n_{i}}(s) v_{n_{i}}(s) d s\right|^{2}\right]^{1 / 2}-1 \geq \frac{c m^{1 / 2}}{4}-1
\end{aligned}
$$

where $c>0$. This contradiction finishes the proof.

From Theorem 6, it follows that every subspace of $\mathcal{R}$ isomorphic to $l_{2}$ is uncomplemented in $K$. However, the following result holds.

Proposition 2. Every subspace $X$ of $\mathcal{R}$ isomorphic to $l_{2}$ contains a subspace complemented in $\mathcal{R}$.

Proof. Let $\left\{x_{n}\right\}_{n=1}^{\infty} \subset X$ be a sequence equivalent to the unit vector basis in $l_{2}$. Arguing as in the proof of Theorem 6, we can find a subsequence $\left\{x_{n_{i}}\right\} \subset\left\{x_{n}\right\}$ which is equivalent in $K$ to a suitable block basis $\left\{u_{i}\right\}_{i=1}^{\infty}$ of the Rademacher functions such that $\left\|u_{i}\right\|_{K} \geq \varepsilon$ and $u_{i} \rightarrow 0$ weakly in $K$. Moreover, we can assume that $\left[x_{n_{i}}\right]$ is complemented in $\mathcal{R}$ if and only if $\left[u_{i}\right]$ is complemented in $\mathcal{R}$. Since $\left\{u_{i}\right\}$ is equivalent to the unit vector basis in $l_{2}$, equivalence (3.1) holds. Let

$$
u_{n}=\sum_{k=m_{n}+1}^{m_{n+1}} a_{k} r_{k}, \quad 1=m_{1}<m_{2}<\cdots, n \in \mathbb{N} .
$$

For any $f=\sum_{n=1}^{\infty} c_{k} r_{k} \in \mathcal{R}$ we set

$$
P f=\sum_{n=1}^{\infty} b_{n}(f) u_{n}, \quad \text { where } \quad b_{n}(f)=\frac{1}{m_{n+1}-m_{n}} \sum_{k=m_{n}+1}^{m_{n+1}} c_{k} .
$$

Then $P g=g$ if $g \in\left[u_{n}\right]$. Moreover, by (3.1) and (1.1),

$$
\begin{aligned}
\|P f\|_{K} & \asymp\left(\sum_{n=1}^{\infty} b_{n}(f)^{2}\right)^{1 / 2}=\left(\sum_{n=1}^{\infty} \frac{1}{\left(m_{n+1}-m_{n}\right)^{2}}\left(\sum_{k=m_{n}+1}^{m_{n+1}} c_{k}\right)^{2}\right)^{1 / 2} \\
& \leq\left(\sum_{n=1}^{\infty} \frac{1}{m_{n+1}-m_{n}} \sum_{k=m_{n}+1}^{m_{n+1}} c_{k}^{2}\right)^{1 / 2} \leq\left(\sum_{k=1}^{\infty} c_{k}^{2}\right)^{1 / 2} \leq C\|f\|_{K} .
\end{aligned}
$$


Thus, $P$ is a bounded projection from $\mathcal{R}$ onto $\left[u_{n}\right]$. By the above observation, this implies that the subspace $\left[x_{n_{i}}\right]$ of $X$ is complemented in $\mathcal{R}$.

4. Structure of Rademacher subspaces in $K_{p}, 1<p<\infty$. Here, we prove Theorem 2. Clearly, it is an immediate consequence of Theorem 3 and the following result.

Theorem 7. Let $1<p<\infty$. Every subspace $X$ of $\mathcal{R}_{p}$ isomorphic to $l_{2}$ is complemented in $K_{p}$.

Proof. Let us prove that for every $x=\sum_{k=1}^{\infty} a_{k} r_{k} \in X$ we have

$$
\|x\|_{K_{p}} \asymp\|x\|_{d}=\left(\sum_{k=1}^{\infty} a_{k}^{2}\right)^{1 / 2},
$$

with constants independent of $x \in X$. In view of (1.1), $\|x\|_{K_{p}} \geq c\|x\|_{d}$ for all $x \in \mathcal{R}_{p}$. Hence, assuming the contrary, we find a sequence $\left\{x_{n}\right\} \subset X$ such that $\left\|x_{n}\right\|_{K_{p}}=1(n=1,2, \ldots)$ and $\left\|x_{n}\right\|_{d} \rightarrow 0$ as $n \rightarrow \infty$.

Since $X$ is isomorphic to $l_{2}$, we may assume that $x_{n} \rightarrow x$ weakly in $K_{p}$ for some $x \in X$. Then, setting $x_{n}=\sum_{k=1}^{\infty} a_{k}^{n} r_{k}, n=1,2, \ldots$, and $x=$ $\sum_{k=1}^{\infty} a_{k} r_{k}$, we see that $\lim _{n \rightarrow \infty} a_{k}^{n}=a_{k}$ for each $k=1,2, \ldots$ On the other hand, $\lim _{n \rightarrow \infty} a_{k}^{n}=0, k=1,2, \ldots$ because $\left\|x_{n}\right\|_{d} \rightarrow 0$, and so $x_{n} \rightarrow 0$ weakly in $K_{p}$. Therefore, applying the Bessaga-Pełczyński Selection Principle, we can find a subsequence $\left\{x_{n_{i}}\right\} \subset\left\{x_{n}\right\}$ and a block basis $\left\{u_{i}\right\}$ of the Rademacher functions such that $\left\|x_{n_{i}}-u_{i}\right\|_{K_{p}} \rightarrow 0$ as $i \rightarrow \infty$, and $\left\{x_{n_{i}}\right\}$ and $\left\{u_{i}\right\}$ are equivalent in $K_{p}$. It is obvious that $\left\|u_{i}\right\|_{K_{p}} \asymp 1(i=1,2, \ldots)$ and $u_{i} \stackrel{w}{\rightarrow} 0$ as $i \rightarrow \infty$ in $K_{p}$. Moreover, by 1.1 ,

$$
\left\|u_{i}\right\|_{d} \leq\left\|x_{n_{i}}-u_{i}\right\|_{d}+\left\|x_{n_{i}}\right\|_{d} \leq C\left\|x_{n_{i}}-u_{i}\right\|_{K_{p}}+\left\|x_{n_{i}}\right\|_{d}
$$

whence $\left\|u_{i}\right\|_{d} \rightarrow 0$ as $i \rightarrow \infty$. But then, by Theorem 3 (b), $\left\{u_{i}\right\}_{i=1}^{\infty}$ contains a subsequence equivalent to the unit vector basis of $c_{0}$. Clearly, this contradicts the assumption, and relation 4.1 is proved.

Recall that the orthogonal projection $\mathcal{P}$ acts boundedly from $L_{p}$ with $1<p<\infty$ onto the closed linear span $\left[r_{n}\right]$ in $L_{p}$. Since the Rademacher functions are equivalent in $L_{p}, 1 \leq p<\infty$, to the unit vector basis of $l_{2}$, from (4.1) it follows that $X$ is a complemented subspace in $\left[r_{n}\right]$. Denote by $R$ a projection from $\left[r_{n}\right]$ onto $X$. Then $S=R \mathcal{P}$ is a bounded projection from $L_{p}$ onto $X$. Moreover, since

$$
\|x\|_{L_{p}} \leq \sup _{0<x \leq 1}\left(\frac{1}{x} \int_{0}^{x}|x(t)|^{p} d t\right)^{1 / p}=\|x\|_{K_{p}} \quad \text { for all } x \in K_{p},
$$

we have

$$
\|S x\|_{K_{p}} \asymp\|S x\|_{L_{p}} \leq C\|S\|_{L_{p} \rightarrow L_{p}}\|x\|_{L_{p}} \leq C_{1}\|\mathcal{P}\|_{L_{p} \rightarrow L_{p}}\|x\|_{K_{p}}
$$

for all $x \in K_{p}$. Thus, $X$ is complemented in $K_{p}$, and the theorem is proved. 
From the Fatou lemma it follows that $K_{p}, 1 \leq p<\infty$, has the Fatou property, i.e., the conditions $f_{n} \in K_{p},\left\|f_{n}\right\|_{K_{p}} \leq C, n=1,2, \ldots, f_{n} \rightarrow f$ a.e. on $[0,1]$ imply that $f \in K_{p}$ and $\|f\|_{K_{p}} \leq C$. Therefore, if $X$ is a subspace of the Rademacher space $\mathcal{R}_{p}, 1 \leq p<\infty$, isomorphic to $c_{0}$, then by the Bessaga-Pełczyński theorem, we can select a block basis $\left\{u_{n}\right\}_{n=1}^{\infty}$ of the Rademacher functions which is equivalent to the unit vector basis in $c_{0}$ and then $K_{p}$ contains the subspace $\tilde{X}_{p}$ consisting of all functions

$$
\left.f=\sum_{n=1}^{\infty} a_{n} u_{n}, \quad \text { where } \quad\left(a_{n}\right) \in l_{\infty} \quad \text { (the series converges a.e. on }[0,1]\right) .
$$

Clearly, $\tilde{X}_{p}$ is isomorphic to $l_{\infty}$. Note that the existence of a bounded projection from $K_{p}$ onto $\left[u_{n}\right] \approx c_{0}$ would imply immediately that we have a bounded projection from $\tilde{X}_{p} \approx l_{\infty}$ onto $\left[u_{n}\right]$, which contradicts the wellknown Phillips-Sobczyk theorem (see [1, Theorem 2.5.5]. Thus, we obtain

COROLlary 2. Every subspace of $\mathcal{R}_{p}, 1 \leq p<\infty$, isomorphic to $c_{0}$ is uncomplemented in $K_{p}$.

5. Rademacher functions in weighted Cesàro spaces. In [6], we have also considered a more general weighted version of Cesàro type spaces, the space $K_{p, w}=K_{p, w}[0,1]$ with the norm

$$
\|f\|_{K_{p, w}}=\sup _{0<x \leq 1}\left(\frac{1}{w(x)} \int_{0}^{x}|f(t)|^{p} d t\right)^{1 / p},
$$

where $1 \leq p<\infty$ and $w$ is a quasi-concave function on $[0,1]$, that is, $w(0)=0, w$ is non-decreasing and $w(x) / x$ is non-increasing on $(0,1]$. Using the equivalence (cf. [6, Theorem 2])

$$
\left\|\sum_{k=1}^{\infty} a_{k} r_{k}\right\|_{K_{p, w}} \asymp\left(\sum_{k=1}^{\infty} a_{k}^{2}\right)^{1 / 2}+\sup _{m \in \mathbb{N}}\left(\frac{2^{-m}}{w\left(2^{-m}\right)}\right)^{1 / p}\left|\sum_{k=1}^{m} a_{k}\right|
$$

and the fact that the restriction of $K_{1, w}$ to any interval $[\delta, 1]$, where $0<$ $\delta<1$, coincides with $L_{1}[\delta, 1]$ (with equivalence of norms), and applying the Dunford-Pettis property of the latter space, we proved in [6, Theorem 5] that the closed linear span $\left[r_{n}\right]$ of the Rademacher functions in $K_{1, w}$ is uncomplemented. The situation is different in the case when $1<p<\infty$. If there is a constant $c>0$ such that

$$
w(t) \geq c t \log _{2}^{p / 2}(2 / t) \quad \text { for all } 0<t \leq 1,
$$

then $\left\{r_{n}\right\}_{n=1}^{\infty}$ is equivalent in $K_{p, w}$ to the unit vector basis of $l_{2}$, and $\left[r_{n}\right]$ is complemented in $K_{p, w}$ (see [6, Theorems 3 and 5]). 
Now, the techniques based on using block bases from this paper allow us to fill the gap in [6] related to the case when condition (5.2) does not hold.

TheOREM 8. If $1<p<\infty$ and condition (5.2) does not hold, then the subspace $\left[r_{n}\right]$ of the Rademacher functions is not complemented in $K_{p, w}$.

Indeed, arguing as in the proof of Theorem 4, we can construct a block basis $\left\{u_{n}\right\}$ of the Rademacher functions equivalent to the unit vector basis of $c_{0}$ such that the closed linear span $\left[u_{n}\right]$ in $K_{p, w}$ is complemented in the subspace $\left[r_{n}\right]$ and not complemented in $K_{p, w}$ (see Corollary 2). Clearly, these facts imply that $\left[r_{n}\right]$ is not complemented in $K_{p, w}$. We omit the details.

\section{References}

[1] F. Albiac and N. J. Kalton, Topics in Banach Space Theory, Grad. Texts in Math. 233, Springer, New York, 2006.

[2] S. V. Astashkin, Rademacher functions in symmetric spaces, Sovrem. Mat. Fundam. Napravl. 32 (2009), 3-161 (in Russian); English transl.: J. Math. Sci. (N. Y.) 169 (2010), 725-886.

[3] S. V. Astashkin, On the geometric properties of Cesàro spaces, Mat. Sb. 203 (2012), no. 4, 61-80 (in Russian); English transl.: Sb. Math. 203 (2012), 514-533.

[4] S. V. Astashkin, M. V. Leıbov and L. Maligranda, Rademacher functions in BMO, Studia Math. 205 (2011), 83-100.

[5] S. V. Astashkin and L. Maligranda, Structure of Cesàro function spaces, Indag. Math. (N.S.) 20 (2009), 329-379.

[6] S. V. Astashkin and L. Maligranda, Rademacher functions in Cesàro type spaces, Studia Math. 198 (2010), 235-247.

[7] S. V. Astashkin and L. Maligranda, Geometry of Cesàro function spaces, Funktsional. Anal. i Prilozhen. 45 (2011), no. 1, 79-83 (in Russian); English transl.: Funct. Anal. Appl. 45 (2011), 64-68.

[8] S. V. Astashkin and L. Maligranda, Structure of Cesàro function spaces: a survey, in: Function Spaces X (Poznań, 2012), Banach Center Publ. 102, Inst. Math., Polish Acad. Sci., 2014, 13-40.

[9] L. V. Kantorovich and G. P. Akilov, Functional Analysis, Nauka, Moscow, 1977 (in Russian); English transl.: Pergamon Press, Oxford, 1982.

[10] B. I. Korenblyum, S. G. Kreĭn and B. Ya. Levin, On certain nonlinear questions of the theory of singular integrals, Dokl. Akad. Nauk SSSR (N.S.) 62 (1948), 17-20 (in Russian).

[11] M. V. Leibov, Geometry of the Function Space BMO, Candidate's Dissertation, Rostov-na-Donu, 1985, 133 pp. (in Russian).

[12] J. Lindenstrauss and L. Tzafriri, Classical Banach Spaces, I. Sequence Spaces, Springer, Berlin, 1977.

[13] J. Lindenstrauss and L. Tzafriri, Classical Banach Spaces, II. Function Spaces, Springer, Berlin, 1979.

[14] W. A. J. Luxemburg and A. C. Zaanen, Some examples of normed Köthe spaces, Math. Ann. 162 (1965/1966), 337-350. 
[15] V. A. Rodin and E. M. Semenov, The complementability of a subspace that is generated by the Rademacher system in a symmetric space, Funktsional. Anal. i Prilozhen. 13 (1979), no. 2, 91-92 (in Russian); English transl.: Funct. Anal. Appl. 13 (1979), no. $2,150-151$.

[16] W. Wnuk, Banach Lattices with Order Continuous Norms, PWN-Polish Sci. Publ., Warszawa, 1999.

[17] A. C. Zaanen, Riesz Spaces II, North-Holland, Amsterdam, 1983.

Sergey V. Astashkin

Department of Mathematics and Mechanics

Samara State University

Acad. Pavlova 1

443011 Samara, Russia

E-mail: astash@samsu.ru
Lech Maligranda

Department of Engineering Sciences and Mathematics Luleå University of Technology SE-971 87 Luleå, Sweden E-mail: lech.maligranda@ltu.se

Received July 20, 2014

Revised version April 18, 2015 
